\title{
Spectroscopic sensing and applications in Silicon Photonics
}

\author{
Eva Ryckeboer, Xiaomin Nie, Ashim Dhakal, Daan Martens, Peter Bienstman, Gunther Roelkens and Roel Baets \\ Photonics Research Group, Ghent University-IMEC \\ Center of Nano- and Biophotonics (NB-photonics) \\ Ghent, Belgium \\ Email: Eva.Ryckeboer@ugent.be
}

\begin{abstract}
We report on miniaturized spectroscopic sensors that are realized using Silicon Photonics technology. This technology relies on CMOS compatible processes to fabricate both Silicon and Silicon-Nitride based photonics integrated circuits. Various spectroscopic sensor designs and applications are discussed.
\end{abstract}

Keywords—Spectroscopy; Silicon photonics; optical sensors

\section{INTRODUCTION}

Optical spectroscopy is a key method to analyze the chemical composition of a sample. It can offer a high specificity as it relies on the unique absorption/Raman spectrum of a given sample. New application areas of optical spectroscopy outside a lab environment have led to a worldwide effort to miniaturize optical spectrometers. In this effort, photonic integration technology has been very important to realize spectrometers that are both portable, robust and low-cost. Silicon photonics is a photonics integration technology that uses CMOS compatible processes to realize reliable photonic integrated circuits (PIC). This way, novel PICs that contain an on-chip spectrometer can be fabricated at low-cost on a wafer-scale. These on-chip spectrometers have shown superior resolution and multiplexing capabilities. Still, it is a challenge to provide the same spectral bandwidth as its bulk optic counterparts. In this paper, we will discuss the key characteristics of Si photonics technology for use in optical spectroscopy. Next to this we will discuss various sensor designs and the associated design challenges. Finally we will discuss two different applications of optical spectrometers based on Si photonics technology.

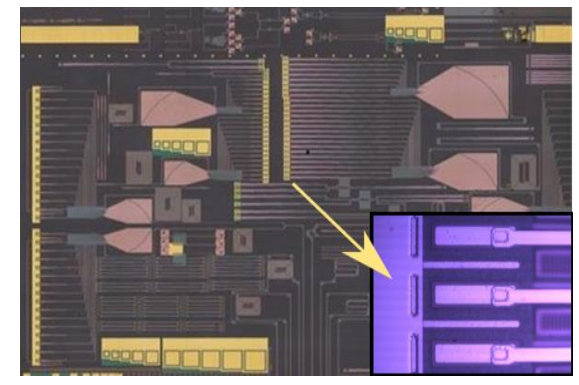

Figure 1: microscope picture of a set of 4 miniature spectrometers integrated on SOI covering a wavelength range from 1510-2300 nm

\section{SILICON PHOTONICS}

Silicon ( $\mathrm{Si}$ ) photonics technology entails two waveguide platforms. The most mature platform is based on silicon-oninsulator (SOI) waveguides and the second platform is based on silicon nitride $\left(\mathrm{Si}_{3} \mathrm{~N}_{4}\right)$ waveguides. The operational wavelength range is limited to $1.1 \mu \mathrm{m}-4 \mu \mathrm{m}$ for SOI based spectrometers. For wavelengths below $1.1 \mu \mathrm{m}$, silicon starts to absorb and beyond $4 \mu \mathrm{m}$, the silicon oxide is absorbing. Therefore, for shorter wavelengths than $1.1 \mu \mathrm{m}$, PICs are developed that use $\mathrm{Si}_{3} \mathrm{~N}_{4}$ as the waveguide layer instead of silicon. Depending on the wavelength range of interest, a different source and detector material is used for the spectrometer. These sources/detectors can be added as discrete units using flip-chip technology or the appropriate materials can be heterogeneously integrated (transfer-printing, bonding). It is still largely a research effort to demonstrate the latter on a wafer-scale. Yet, as silicon is an efficient detector below $1.1 \mu \mathrm{m}$, highly performant CMOS imager wafers have the potential to enable wafer-scale detectors for $\mathrm{Si}_{3} \mathrm{~N}_{4}$-based spectroscopic applications.

Much progress has been made in both waveguide platforms to realize optical wavelength demultiplexers, a critical component for dispersive optical spectroscopy. Common types of demultiplexers such as arrayed waveguide grating (AWG) and planar concave grating (PCG), ring resonator arrays and Mach-Zehnder based lattice filters have been demonstrated in various wavelength ranges spanning the visible to the MID-IR [1]. Also Fourier transform infrared spectrometers (FTIR or FTS) have been realized in Si photonics technology [2]. A spatially varying interferogram is captured in this case, therefore the spectrometer doesn't need any moving parts. Generally, the performance of the $\mathrm{Si}_{3} \mathrm{~N}_{4}$ based spectrometers is better than for SOI in terms of insertion loss and optical crosstalk. This is due to the reduced refractive index contrast of the $\mathrm{Si}_{3} \mathrm{~N}_{4}$ platform compared to SOI, which lowers the sensitivity to phase noise and sidewall roughness.

Also the sample interface can be implemented using $\mathrm{Si}$ photonics technology. In this case the evanescent field of a waveguide is used to interact with the sample. For medium to high refractive index contrast material platform such as SOI and $\mathrm{Si}_{3} \mathrm{~N}_{4}$, this evanescent field is located within $30-100 \mathrm{~nm}$ of the surface of the waveguide. Therefore, molecules or chemical interactions that are present close to the waveguide can be 
readily measured. To increase the interaction length with the sample, a longer waveguide or resonating structures can be used.

The strong waveguide dispersion of both $\mathrm{SOI}$ and $\mathrm{Si}_{3} \mathrm{~N}_{4} \mathrm{PICs}$ and the limited availability of integrated broadband sources, leads to challenges in making the spectrometer broadband in operation. Therefore, spectrometers in Si photonics technology typically target specific spectral features or combine multiple spectrometers on a single chip, each covering a separate wavelength range [3].

\section{SENSOR DESIGN AND APPLICATIONS}

Optical spectrometers can be implemented as optical sensors that directly measure the Raman or absorption spectrum of a sample or as a read-out unit of an optical sensor.

\section{A. On-chip Raman spectroscopy}

Raman spectroscopy is a powerful technique to identify and quantify the number of molecules in a sample thanks to the many and distinct features in typical Raman spectra. Still, Raman scattering is an extremely weak effect. Therefore, advanced sources, detectors and techniques such as surface-enhanced Raman scattering (SERS) are needed to enhance the signal quality. State-of-the-art confocal microscopes are commonly used for Raman spectroscopy. Yet, these are large and costly devices that have limitations when it comes to very thin layers of materials. This is because of the limited interaction length $(<$ $1 \mu \mathrm{m})$ with the material of interest. When using single-mode waveguides to evanescently excite and collect the Raman signals, the Raman signal is strongly enhanced thanks to the field enhancement effect near a high index contrast waveguide, moreover the interaction length can easily be increased. Successful on-chip Raman sensing experiments have been shown with $\mathrm{Si}_{3} \mathrm{~N}_{4}$ chips (e.g. DNA hybridization, monolayer detection). Yet, the waveguides themselves also contribute to the measured Raman signal. This background is strong and generates shot-noise that can reduce the detection limits [4].

\section{B. On-chip absorption spectroscopy}

Light absorption by molecules is very strong in the midinfrared region $(3-10 \mu \mathrm{m})$. At present, this range is not easily accessed with Si photonics technology due to the lack of cheap sources and detectors. When probing in the near infrared, more sources and detectors are available and we can detect the overtones of the fundamental absorption features. Therefore, $\mathrm{Si}$ photonics has been successfully used in this range. In on-chip absorption spectroscopy, Beer-Lambert's law is used to describe the attenuation of the evanescent field with absorption in the cladding. The waveguide transmission $T$ is altered after a distance of $L$ by the losses in both waveguide $\left(\alpha_{\mathrm{wg}}\right)$ and cladding $\left(\alpha_{s}\right)$ as: $T=e^{-\left(\Gamma \alpha_{s}+\alpha_{w g}\right) \mathrm{L}}$. The fill factor $\Gamma$ is the fraction of the optical mode that extends into the cladding. The waveguide length can be optimized to $\mathrm{L}=1 /\left(\Gamma \alpha_{s}+\alpha_{w g}\right)$. For best operation, a low-loss waveguide with a large fill factor is needed. For SOI based circuits, the sidewall roughness of the waveguides gives rise to distributed backscattering. This reflects in the transmission spectrum as a dense ripple which is sensitive to small environmental changes. This backscattering effect should therefore be minimized.

\section{Spectrometer as sensor interrogator}

Optical sensors that yield a shift in spectral response due to the presence of a certain physical (e.g. temperature, humidity, pressure) or chemical effect (e.g. binding event) are very powerful. Rather than a small amplitude change which can result from environmental fluctuations as in Raman/absorption spectroscopy, a frequency change should be detected. Here optical spectrometers can be used as sensor interrogators. Key applications are refractive index sensors, fiber Bragg grating interrogators and pressure sensors. Here Si photonics offers the advantage of a low-cost read-out unit that can multiplex many sensors as the footprint of both the sensor and spectrometer can be very small $\left(<0.5 \mathrm{~mm}^{2}\right)$.

\section{APPLICATIONS}

\section{A. On-chip glucose absorption spectroscopy}

Diabetes is a serious health condition that affects nearly 1 out of 11 people worldwide. For patients it is important to track their glucose levels throughout the day. Such a glucose monitor was investigated with Si photonics technology. The key challenge in this application is the strong water absorption compared to the weak glucose absorption at physiological concentrations. Here a spectrometer with a signal-to-noise ratio of $40 \mathrm{~dB}$ is required. Still, research has shown that is possible to detect aqueous glucose in the relevant concentration ranges using a miniature spectrometer that is so small that it can be implanted [1].

\section{B. Lab-on-chip Tuberculosis detection}

Current diagnostic tests for Tuberculosis (TB) are time consuming and expensive. An alternative low-cost point of care sensor to detect TB in urine is developed using Si photonics technology. The sensor is a Mach-Zehnder interferometer (MZI) that is coupled to an AWG. When a biomarker for TB (LAM) is present in urine, the spectral response of the MZI shifts in frequency which is resolved by the AWG. To allow for a very cheap, disposable sensor, flood illumination and detection in a separate read-out unit is used. Successful detection of low concentrations of the biomarker $(250 \mathrm{pg} / \mathrm{mL}$ LAM) with this very sensitive device proofs the feasibility of this approach [5].

\section{ACKNOWLEDGMENT}

The authors would like to acknowledge ERC-InSpectra Advanced Grant, FP7-Pocket and Methusalem (Smart Photonics Chips) for their support.

\section{REFERENCES}

[1] Subramanian, Ananth, et al. "Silicon and silicon nitride photonic circuits for spectroscopic sensing on-a-chip." Photonics Research 3.5 (2015).

[2] Velasco, Aitor V., et al. "High-resolution Fourier-transform spectrometer chip with microphotonic silicon spiral waveguides." Optics letters 38.5 (2013): 706-708.

[3] Ryckeboer, Eva, et al. "Silicon-on-insulator spectrometers with integrated GaInAsSb photodiodes for wide-band spectroscopy from 1510 to 2300 nm." Optics express 21.5 (2013).

[4] Dhakal, Ashim, et al. "Silicon Nitride Background in Nanophotonic Waveguide Enhanced Raman Spectroscopy." Materials 10.2 (2017)

[5] Geers, Bart, et al."Pocket - point-of-care system for the label free detection of bacterial pathogens", Compamed/Medica tradeshow (2016) 